Review began 11/07/2021 Review ended 01/30/2022 Published 02/07/2022

(๑) Copyright 2022 Lin et al. This is an open access article distributed under the terms of the Creative Commons Attribution License CC-BY 4.0. which permits unrestricted use, distribution, and reproduction in any medium, provided the original author and source are credited.

\section{Southern California Pediatric and Adolescent Cancer Survivorship (SC-PACS): Establishing a Multi-Institutional Childhood, Adolescent, and Young Adult Cancer Survivorship Consortium in Southern California}

Carol Lin ${ }^{1}$, Nicole Baca ${ }^{2}$, Christine Yun ${ }^{1}$, Saro Armenian ${ }^{3,4}$, David R. Freyer ${ }^{5,} 6$, Fataneh Majlessipour ${ }^{2}$ , Lisa Mueller ${ }^{7}$, Dennis J. Kuo ${ }^{8,9}$, Jacqueline Casillas ${ }^{10,11}$, Keri Zabokrtsky ${ }^{12}$, Louis Ehwerhemuepha ${ }^{13}$, Lilibeth Torno 14

\begin{abstract}
1. Department of Pediatric Oncology, Hyundai Cancer Institute, Children's Hospital of Orange County, Orange, USA 2. Department of Pediatrics, Samuel-Oschin Comprehensive Cancer Institute, Cedars-Sinai Medical Center, Los Angeles, USA 3. Department of Pediatrics, City of Hope, Duarte, USA 4. Department of Population Sciences, City of Hope, Duarte, USA 5. Department of Pediatric Oncology, Cancer and Blood Disease Institute, Children's Hospital Los Angeles, Los Angeles, USA 6. Department of Pediatric Oncology, Keck School of Medicine, University of Southern California, Los Angeles, USA 7. Division of Pediatric Hematology and Oncology, Department of Pediatrics, Kaiser Permanente Medical Center, Los Angeles, USA 8. Division of Pediatric Hematology-Oncology, University of California San Diego, San Diego, USA 9. Division of Pediatric Hematology-Oncology, Rady Children's Hospital San Diego, San Diego, USA 10. Division of Hematology and Oncology, Department of Pediatrics, University of California Los Angeles, Los Angeles, USA 11. Department of Pediatrics, Jonathan Jaques Children's Cancer Institute, Miller's Children's Hospital, Long Beach, USA 12. Research Institute, Children's Hospital of Orange County, Orange, USA 13. Department of Computational Research, Children's Hospital of Orange County, Orange, USA 14. Department of Pediatric Oncology, Hyundai Cancer Institute, Children's Hospital of Orange County, Orange, USA
\end{abstract}

Corresponding author: Lilibeth Torno, ltorno@choc.org

\title{
Abstract
}

\section{Introduction}

Given their risk for late effects and early mortality, childhood/adolescent cancer survivors (CACSs) should receive longitudinal monitoring and care. The Southern California Pediatric and Adolescent Cancer Survivorship (SC-PACS) consortium was established in February 2017 to combine resources and expertise across seven participating survivorship programs. Its over-arching objective is to address the unique needs of its demographically diverse CACS population through collaborative survivorship research and care initiatives. The first SC-PACS study was an assessment of survivorship needs and evaluation of current services as reported by CACSs and their parents/primary care givers (PPCGs) receiving survivorship care at consortium sites.

\section{Methods}

As an initial investigation, a cross-sectional survey for CACSs and their parents/primary care givers was conducted. The goal was to enroll 10 CACSs and 10 PPCGs from each of the seven institutions (total of 140 participants). The eligibility criteria for CACSs were age $\geqslant 13$ years at the time of enrollment, $>2$ years from the end of treatment, sufficient cognitive function to complete the survey, and English or Spanish language proficiency. For CACSs $<13$ years old, their PPCGs completed the survey. This was a convenience sample using frequencies and proportions to describe participant characteristics and survey responses, which were entered into a Research Electronic Data Capture (REDCap) database.

\section{Results}

Across the consortium, of the recruitment target of 140 participants (CACSs, $n=70$; PPCGs, $n=70$ ), 127 (90.7\%) participants were enrolled. Of the 127 participants enrolled, 65 (51.2\%) were CACSs and 62 (48.8\%) were PPCGs. The majority of participants were female (51.2\%), were Hispanic (62.2\%), spoke English as the primary language at home (57.5\%), and were diagnosed between one to four years of age (45.7\%). Information considered most important by both CACSs and PPCGs was related to cancer diagnosis (90.8\%) and future risks as a result of cancer treatment received (98.0\%). Overall, 78\% of CACSs and PPCGs found the survivorship information (treatment summary) useful, and $83 \%$ felt that they received the right amount of information about their cancer.

\section{Conclusion}

Our aim was to obtain baseline data that would characterize our CACS population, inform consortium priorities, and establish a collaborative research platform. The ultimate goal of the consortium is to develop a comprehensive survivorship care approach that addresses the most important needs of cancer survivors in our catchment area and promotes best practice interventions. Future plans are to expand the needs 


\section{Cureus}

assessment survey to obtain a wider representation of the survivor population at SC-PACS institutions, helping create strategies to improve cancer-specific education, delivery of treatment summary, and access to community resources for this demographically and socioeconomically diverse population.

Categories: Pediatrics, Oncology

Keywords: survivorship needs, survivorship consortium, childhood/adolescent cancer survivors, pediatric cancer, cancer survivorship

\section{Introduction}

Due to remarkable progress in the treatment of cancer among children and adolescents, their aggregate fiveyear survival now exceeds $84 \%$ [1-3]. However, large cohort and population-based studies have documented the high burden of morbidity and mortality associated with cancer treatment at a young age [4-10]. These studies have included mostly non-Hispanic white participants and few racial and ethnic minorities [11-14]. These underrepresented populations of survivors may have different outcomes and needs that have been understudied to date. With the increasing diversity of the United States [15], cancer survivorship programs must ensure their childhood/adolescent cancer survivors (CACSs) are appropriately represented in research studies and have access to culturally competent care. The racial, ethnic, and sociocultural diversity of southern California's population makes it an ideal environment to gain insight into the most pressing concerns for CACSs living in this region and beyond.

Consequently, the Southern California Pediatric and Adolescent Cancer Survivorship (SC-PACS) consortium was established in February 2017 with the over-arching objective of addressing the unique needs of its demographically diverse CACS population through collaborative survivorship research and care initiatives. The demographic reach of the SC-PACS consortium encompasses Los Angeles, San Diego, and contiguous counties, a region accounting for approximately $40 \%$ of the California population.

The first SC-PACS study was an assessment of survivorship needs and evaluation of current services as reported by CACSs and their parents/primary care givers (PPCGs) receiving survivorship care at consortium sites. Our goal was to obtain baseline data that would characterize our CACS population, inform consortium priorities, and establish a collaborative research platform.

\section{Materials And Methods}

The consortium includes seven cancer treatment centers for children and adolescents, including CedarsSinai Medical Center (Los Angeles, CA), Children's Hospital Los Angeles (Los Angeles, CA), Children's Hospital of Orange County (Orange, CA), City of Hope (Duarte, CA), Kaiser Permanente Medical Center (Los Angeles, CA), Rady Children's Hospital San Diego (San Diego, CA), and University of California, Los Angeles/Miller Children's and Women's Hospital (Long Beach, CA). Cancer survivorship specialists at each site include oncologists, advanced practice providers, and research/case coordinators (institutional characteristics are given in Table 1). 


\section{Cureus}

\begin{tabular}{|c|c|c|c|c|c|c|c|}
\hline \multicolumn{8}{|l|}{ Comprehensive Long-Term Survivorship Program } \\
\hline Type of institution & $\mathrm{CHOC}$ & $\mathrm{COH}$ & CHLA & $\mathrm{KP}$ & RCHSD & UCLA/Miller & Cedars \\
\hline Children's hospital & $\mathrm{x}$ & & $\mathrm{x}$ & & $\mathrm{x}$ & $x$ & \\
\hline Cancer centers or university hospital & & $\mathrm{x}$ & $\mathrm{x}$ & & $\mathrm{x}$ & & \\
\hline Pediatric hospital/unit with adult hospital & & & & & & $\mathrm{x}$ & $\mathrm{x}$ \\
\hline Other & & & & $\mathrm{x}$ & & & \\
\hline \multicolumn{8}{|l|}{ Survivorship program clinical characteristics } \\
\hline Upper age limit < 21 years & & & $\mathrm{x}$ & & $\mathrm{x}$ & $x$ & \\
\hline Upper age limit $\leq 26$ years & $\mathrm{x}$ & & & & & & $x$ \\
\hline No upper age limit & & $\mathrm{X}$ & & $\mathrm{x}$ & & & \\
\hline When are patients referred? How many years off therapy? & $4-5$ & 2 & $1-2$ & $1-2$ & 2 & $1-2$ & \\
\hline How often are they seen in a disease-specific clinic? & Annual & Annual & Annual & Annual & Every 2 years & Annual & Annual \\
\hline Dedicated childhood cancer survivorship clinic & $\mathrm{x}$ & $\mathrm{x}$ & $\mathrm{x}$ & $\mathrm{x}$ & $\mathrm{x}$ & $\mathrm{x}$ & \\
\hline Provide comprehensive treatment summaries & $\mathrm{X}$ & $\mathrm{X}$ & $\mathrm{X}$ & $X$ & $\mathrm{X}$ & $\mathrm{X}$ & $\mathrm{X}$ \\
\hline Multidisciplinary clinic for childhood cancer survivors & $\mathrm{x}$ & $\mathrm{x}$ & $\mathrm{x}$ & $\mathrm{x}$ & $\mathrm{x}$ & $\mathrm{x}$ & \\
\hline Dedicated advanced practice practitioner & $\mathrm{X}$ & $\mathrm{x}$ & $\mathrm{x}$ & $\mathrm{X}$ & $\mathrm{x}$ & $\mathrm{x}$ & \\
\hline Formal transition program to adult survivorship program & & N/A & $\mathrm{X}$ & & $\mathrm{X}$ & & $\mathrm{x}$ \\
\hline
\end{tabular}

\section{TABLE 1: Institutional characteristics}

CHOC, Children's Hospital of Orange County; $\mathrm{COH}$, City of Hope; CHLA, Children's Hospital Los Angeles; KP, Kaiser Permanente Medical Center; RCHSD, University of California, San Diego/Rady Children's Hospital San Diego; UCLA/Miller, University of California, Los Angeles/Miller Children's and Women's Hospital; Cedars, Cedars-Sinai Medical Center; N/A, not applicable

Since its inception, SC-PACS members have met semi-annually in person (until the COVID-19 pandemic), supplemented by monthly teleconferences. Each site's institutional characteristics, clinical service, and research capabilities were outlined initially. Due to its geographically central location, Children's Hospital of Orange County functions as the consortium's administrative hub and governing site. A Data Use Agreement was finalized in February 2018.

We conducted a multi-center, cross-sectional, survey-based study of CACSs and their PPCGs. The goal was to enroll 10 CACSs and 10 PPCGs from each of the seven institutions (total of 140 participants). The eligibility criteria for CACSs were age $\geqslant 13$ years at the time of enrollment, $>2$ years from the end of treatment, sufficient cognitive function to complete the survey, and English or Spanish language proficiency. In this cohort, only the CACSs were surveyed. For CACSs $<13$ years old, their PPCGs completed the survey. All participants provided written informed consent or assent. The study was approved by each site's Institutional Review Board (IRB) prior to enrollment of its first participant (Children's Hospital of Orange County In-House IRB \#1709102).

Participants were approached during a clinic visit and given a paper questionnaire to complete prior to discharge. The questionnaire was intentionally created for the purpose of this study and was not adapted from a previous, validated tool. Survey items included demographics and 33 questions evaluating the importance and usefulness of health information gained during their survivorship clinic encounters. Feasibility was defined as enrollment of $\geqslant 80 \%$ of participants who were approached at each site. This was a convenience sample using frequencies and proportions to describe participant characteristics and survey responses, which were entered into a Research Electronic Data Capture (REDCap) database (Vanderbilt University, Nashville, TN).

\section{Results}

Across the consortium, of the recruitment target of 140 participants (CACSs, $n=70$; PPCGs, $n=70$ ), 127 (90.7\%) participants were enrolled. At four sites, 20 participants were approached and enrolled; at two sites, 19 and 6 participants, respectively, were approached and enrolled; and at one site, 22 participants were 


\section{Cureus}

approached and enrolled. A total of 65 (51.2\%) participants were CACSs and 62 (48.8\%) were PPCGs.

The majority of participants were female (51.2\%), were Hispanic (62.2\%), spoke English as the primary language at home (57.5\%), and were diagnosed between one and four years of age $(45.7 \%)$ (survivor characteristics are given in Table 2).

\begin{tabular}{|c|c|c|}
\hline Characteristics & Levels & $\mathbf{n}(\%)$ \\
\hline \multirow{2}{*}{ Gender } & Female & $65(51.2)$ \\
\hline & Male & $62(48.8)$ \\
\hline \multirow{4}{*}{ Ethnicity } & Hispanic or Latino & $79(62.2)$ \\
\hline & Not Hispanıc or Latıno & $44(34.6)$ \\
\hline & Missing responses & $3(2.4)$ \\
\hline & I would rather not say & $1(0.8)$ \\
\hline \multirow{8}{*}{ Race } & White & $56(44.1)$ \\
\hline & Asian or Pacific Islander & $8(6.3)$ \\
\hline & Black & $3(2.4)$ \\
\hline & American Indian/Alaskan Native & $3(2.4)$ \\
\hline & Other Asian or Pacific Islander & $11(8.7)$ \\
\hline & Missing responses & $28(22.0)$ \\
\hline & Multiple responses & $9(7.1)$ \\
\hline & I would rather not say & $9(7.1)$ \\
\hline \multirow{5}{*}{ Age at diagnosis } & <1 year of age & $13(10.2)$ \\
\hline & 1 to 4 years of age & $58(45.7)$ \\
\hline & 5 to 9 years of age & $32(25.2)$ \\
\hline & 10 to 14 years of age & $12(9.4)$ \\
\hline & 15 to 19 years of age & $12(9.4)$ \\
\hline \multirow{11}{*}{ Cancer diagnosis } & Leukemia & $63(49.6)$ \\
\hline & Lymphoma & $16(12.6)$ \\
\hline & Wilms tumor & $11(8.7)$ \\
\hline & Bone tumor & $6(4.7)$ \\
\hline & Brain tumor & $5(3.9)$ \\
\hline & Neuroblastoma & $5(3.9)$ \\
\hline & Germ cell tumor & $4(3.1)$ \\
\hline & Soft tissue sarcoma & $3(2.4)$ \\
\hline & Other (describe) & $12(9.4)$ \\
\hline & do not know & $1(0.8)$ \\
\hline & Missing responses & $1(0.8)$ \\
\hline \multirow{4}{*}{ Primary language spoken at home } & English & $73(57.5)$ \\
\hline & Spanish & $29(22.8)$ \\
\hline & Arabic & $1(0.8)$ \\
\hline & IVandarın/Cantonese & $1(0.8)$ \\
\hline
\end{tabular}




\section{Cureus}

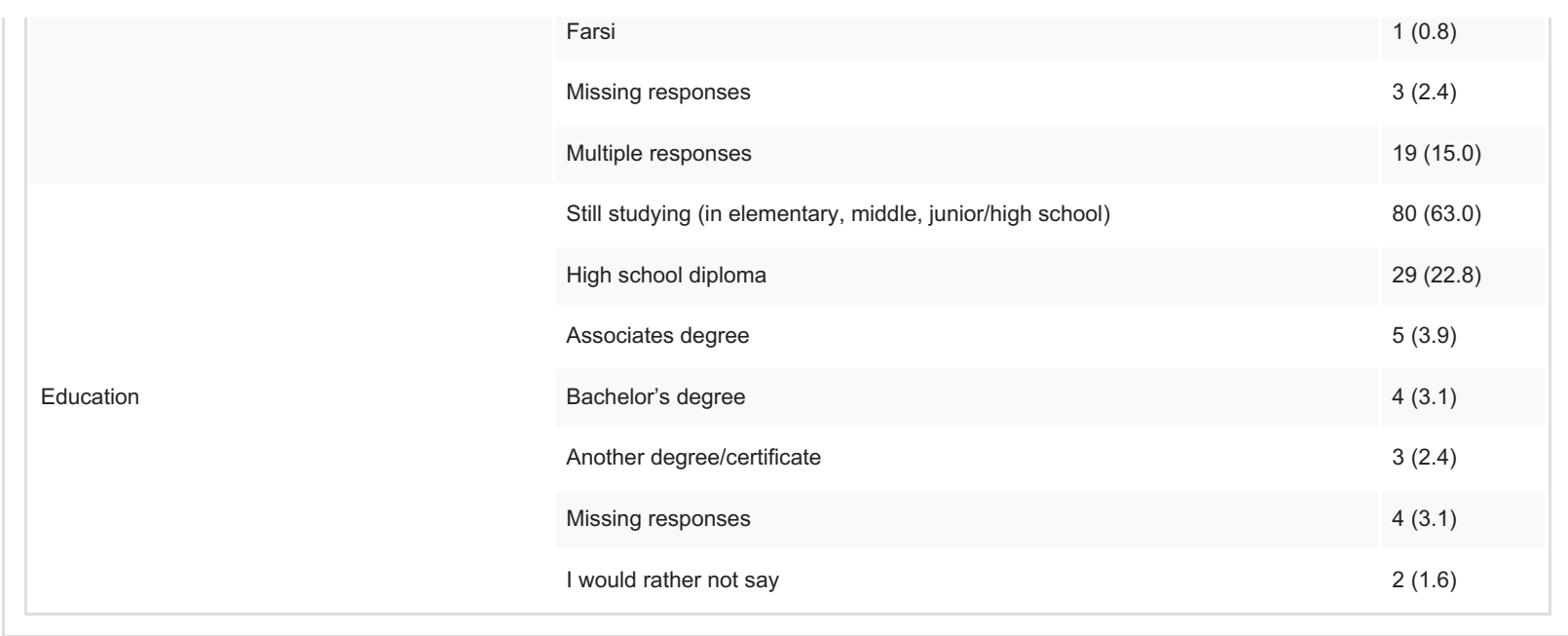

TABLE 2: Survivor characteristics

Leukemia (63\%) and lymphoma (16\%) were the most common diagnoses. Information considered most important by both CACSs and PPCGs was related to cancer diagnosis (90.8\%) and future risks of treatment (98.0\%). There was a slight preference for electronic method of delivery of medical information over paper for both CACSs (38.5\% vs. 35.4\%) and PPCGs (43.5\% vs. 33.9\%). Access and resources for psychosocial support were also deemed important by both groups (survey responses are given in Table 3).

\begin{tabular}{|c|c|c|c|}
\hline Variables & Levels & $\begin{array}{l}\text { CACS } \\
\mathrm{n}(\%)\end{array}$ & $\begin{array}{l}\text { PPCG* } \\
\text { n (\%) }\end{array}$ \\
\hline \multirow{4}{*}{ Information about cancer, its treatment, and future health risks } & Very important & $\begin{array}{l}59 \\
(90.8)\end{array}$ & $\begin{array}{l}61 \\
(98.4)\end{array}$ \\
\hline & $\begin{array}{l}\text { Somewhat } \\
\text { important }\end{array}$ & $\begin{array}{l}5 \\
(7.7)\end{array}$ & $0(0.0)$ \\
\hline & $\begin{array}{l}\text { I would rather not } \\
\text { say }\end{array}$ & $\begin{array}{l}1 \\
(1.5)\end{array}$ & $0(0.0)$ \\
\hline & Missing responses & $\begin{array}{l}0 \\
(0.0)\end{array}$ & $1(1.6)$ \\
\hline \multirow{4}{*}{ Information about getting a second cancer in the future } & Very important & $\begin{array}{l}54 \\
(83.1)\end{array}$ & $\begin{array}{l}61 \\
(98.4)\end{array}$ \\
\hline & $\begin{array}{l}\text { Somewhat } \\
\text { important }\end{array}$ & $\begin{array}{l}8 \\
(12.3)\end{array}$ & $0(0.0)$ \\
\hline & No opinion & $\begin{array}{l}1 \\
(1.5)\end{array}$ & $1(1.6)$ \\
\hline & $\begin{array}{l}\text { I would rather not } \\
\text { say }\end{array}$ & $\begin{array}{l}2 \\
(3.1)\end{array}$ & $0(0.0)$ \\
\hline \multirow{5}{*}{ Information about how to check for symptoms that cancer recurs } & Very important & $\begin{array}{l}57 \\
(87.7)\end{array}$ & $\begin{array}{l}62 \\
(100.0)\end{array}$ \\
\hline & $\begin{array}{l}\text { Somewhat } \\
\text { important }\end{array}$ & $\begin{array}{l}7 \\
(10.8)\end{array}$ & $0(0.0)$ \\
\hline & No opinion & $\begin{array}{l}1 \\
(1.5)\end{array}$ & $0(0.0)$ \\
\hline & Very important & $\begin{array}{l}41 \\
(63.1)\end{array}$ & $\begin{array}{l}56 \\
(90.3)\end{array}$ \\
\hline & $\begin{array}{l}\text { Somewhat } \\
\text { important }\end{array}$ & $\begin{array}{l}14 \\
(21.5)\end{array}$ & $3(4.8)$ \\
\hline
\end{tabular}




\section{Cureus}

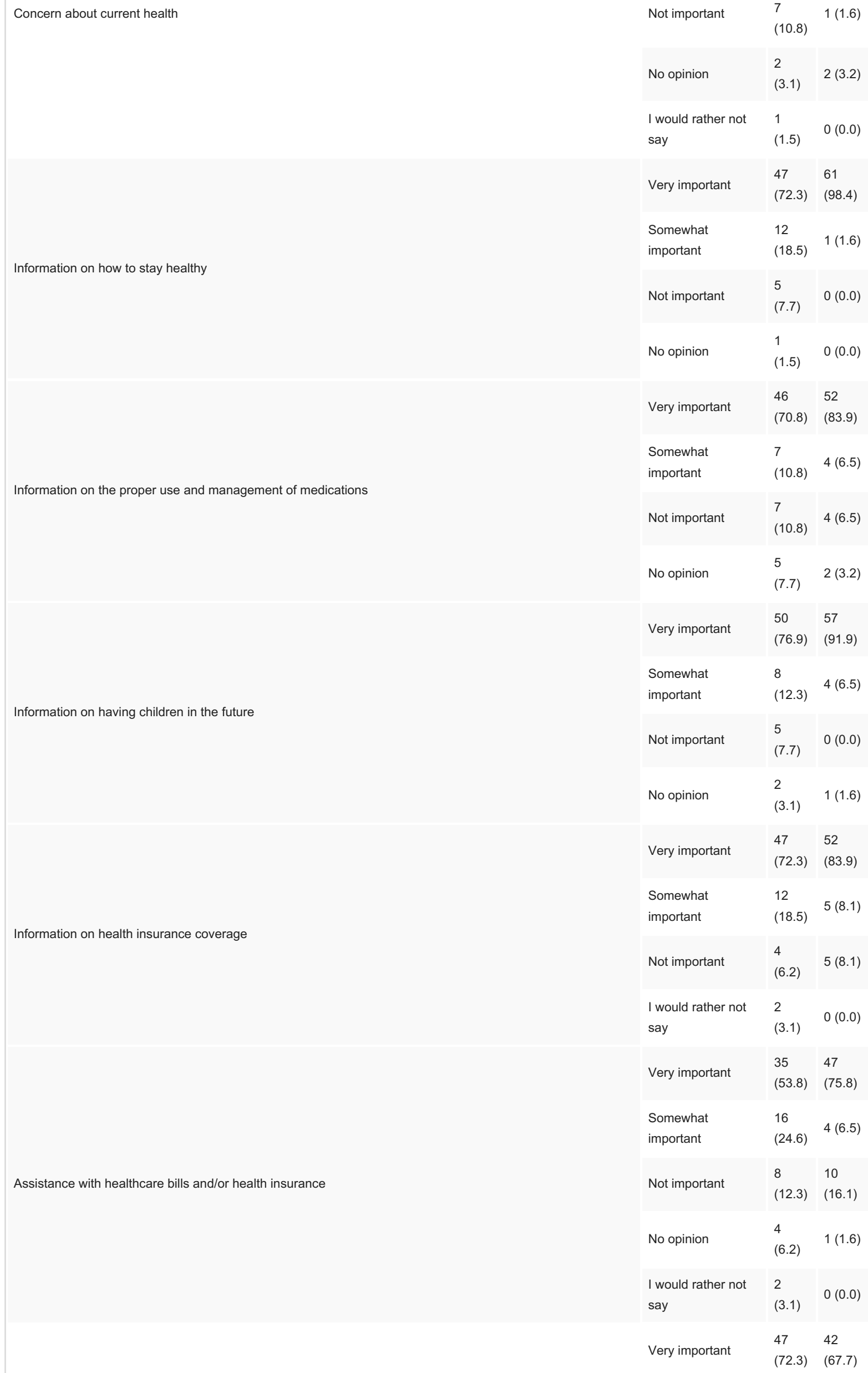




\section{Cureus}

Information on how the healthcare system works

Access to internet sites to learn more about survivorship

Access to peer or survivor support groups

Access to camps or other retreats for cancer survivors

Information on where to seek help for managing feelings and learning coping strategies

Access to practical support (scholarships, jobs, transportation, etc.)

\begin{tabular}{|c|c|c|}
\hline $\begin{array}{l}\text { Somewhat } \\
\text { important }\end{array}$ & $\begin{array}{l}12 \\
(18.5)\end{array}$ & $\begin{array}{l}12 \\
(19.4)\end{array}$ \\
\hline Not important & $\begin{array}{l}4 \\
(6.2)\end{array}$ & $6(9.7)$ \\
\hline No opinion & $\begin{array}{l}0 \\
(0.0)\end{array}$ & $2(3.2)$ \\
\hline $\begin{array}{l}\text { I would rather not } \\
\text { say }\end{array}$ & $\begin{array}{l}2 \\
(3.1)\end{array}$ & $0(0.0)$ \\
\hline Very important & $\begin{array}{l}32 \\
(49.2)\end{array}$ & $\begin{array}{l}48 \\
(77.4)\end{array}$ \\
\hline $\begin{array}{l}\text { Somewhat } \\
\text { important }\end{array}$ & $\begin{array}{l}24 \\
(36.9)\end{array}$ & $\begin{array}{l}12 \\
(19.4)\end{array}$ \\
\hline Not important & $\begin{array}{l}4 \\
(6.2)\end{array}$ & $1(1.6)$ \\
\hline No opinion & $\begin{array}{l}5 \\
(7.7)\end{array}$ & $0(0.0)$ \\
\hline Missing response & $\begin{array}{l}0 \\
(0.0)\end{array}$ & $1(1.6)$ \\
\hline Very important & $\begin{array}{l}22 \\
(33.8)\end{array}$ & $\begin{array}{l}21 \\
(33.9)\end{array}$ \\
\hline $\begin{array}{l}\text { Somewhat } \\
\text { important }\end{array}$ & $\begin{array}{l}19 \\
(29.2)\end{array}$ & $\begin{array}{l}36 \\
(58.1)\end{array}$ \\
\hline Not important & $\begin{array}{l}15 \\
(23.1)\end{array}$ & $4(6.5)$ \\
\hline No opinion & $\begin{array}{l}9 \\
(13.8)\end{array}$ & $1(1.6)$ \\
\hline Very important & $\begin{array}{l}26 \\
(40.0)\end{array}$ & $\begin{array}{l}36 \\
(58.1)\end{array}$ \\
\hline $\begin{array}{l}\text { Somewhat } \\
\text { important }\end{array}$ & $\begin{array}{l}13 \\
(20.0)\end{array}$ & $\begin{array}{l}19 \\
(30.6)\end{array}$ \\
\hline Not important & $\begin{array}{l}18 \\
(27.7)\end{array}$ & $6(9.7)$ \\
\hline No opinion & $\begin{array}{l}8 \\
(12.3)\end{array}$ & $1(1.6)$ \\
\hline Very important & $\begin{array}{l}26 \\
(40.0)\end{array}$ & $\begin{array}{l}43 \\
(69.4)\end{array}$ \\
\hline $\begin{array}{l}\text { Somewhat } \\
\text { important }\end{array}$ & $\begin{array}{l}19 \\
(29.2)\end{array}$ & $\begin{array}{l}14 \\
(22.6)\end{array}$ \\
\hline Not important & $\begin{array}{l}14 \\
(21.5)\end{array}$ & $4(6.5)$ \\
\hline No opinion & $\begin{array}{l}5 \\
(7.7)\end{array}$ & $0(0.0)$ \\
\hline Missing responses & $\begin{array}{l}1 \\
(1.5)\end{array}$ & $1(1.6)$ \\
\hline Very important & $\begin{array}{l}43 \\
(66.2)\end{array}$ & $\begin{array}{l}46 \\
(74.2)\end{array}$ \\
\hline $\begin{array}{l}\text { Somewhat } \\
\text { important }\end{array}$ & $\begin{array}{l}16 \\
(24.6)\end{array}$ & $\begin{array}{l}10 \\
(16.1)\end{array}$ \\
\hline Not important & 6 & $4(6.5)$ \\
\hline
\end{tabular}




\section{Cureus}

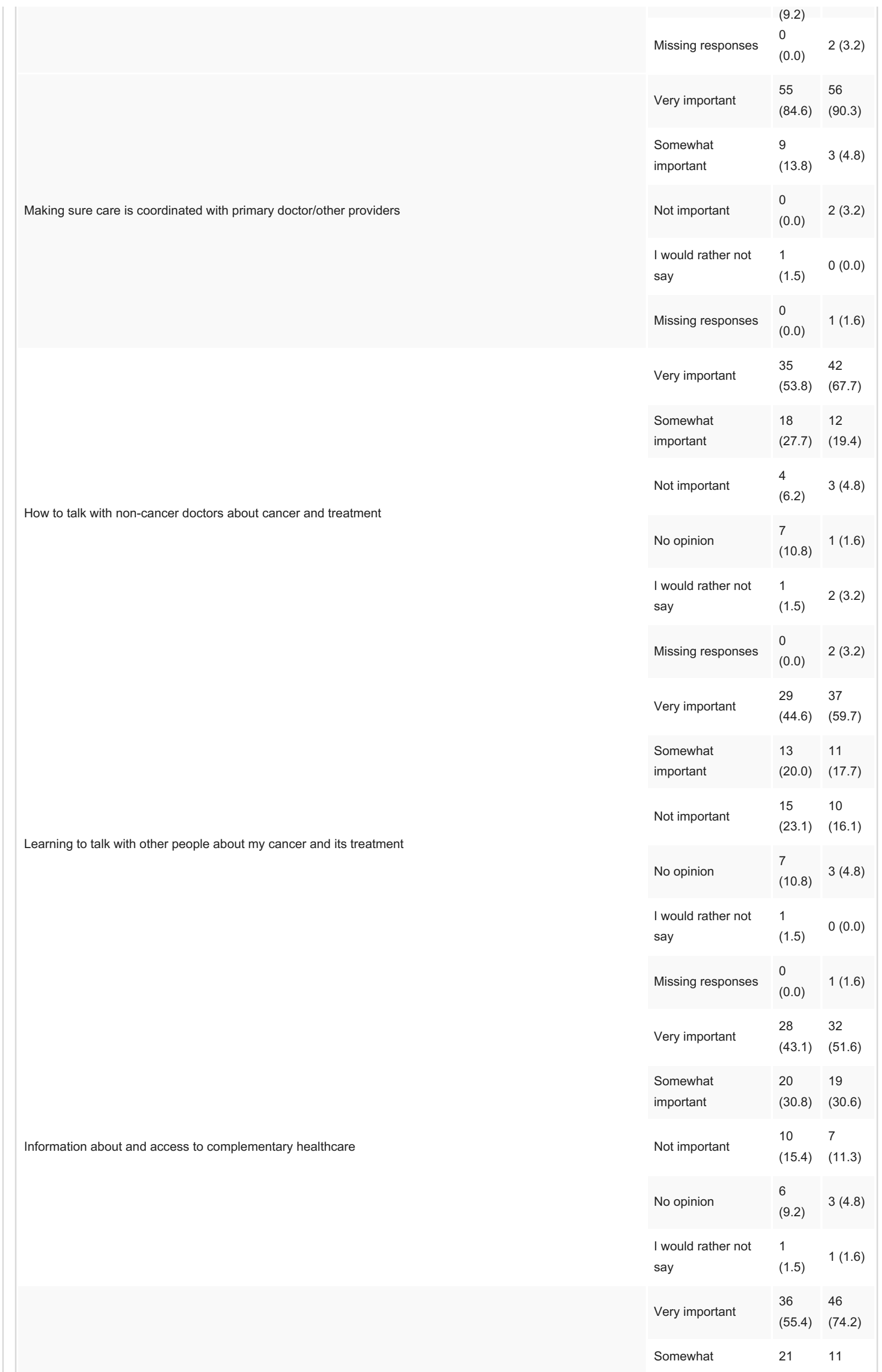




\section{Cureus}

\begin{tabular}{|c|c|c|c|}
\hline \multirow{3}{*}{ Learning about ways to help other patients or families } & & \\
\hline & Not important & $\begin{array}{l}3 \\
(4.6)\end{array}$ & $3(4.8)$ \\
\hline & No opinion & $\begin{array}{l}5 \\
(7.7)\end{array}$ & $2(3.2)$ \\
\hline \multirow{6}{*}{ Information regarding survivorship that was given during your previous survivorship clinic visit(s)? } & Yes, a lot & $\begin{array}{l}19 \\
(29.2)\end{array}$ & $\begin{array}{l}32 \\
(51.6)\end{array}$ \\
\hline & Yes, a little & $\begin{array}{l}31 \\
(47.7)\end{array}$ & $\begin{array}{l}13 \\
(21.0)\end{array}$ \\
\hline & $\begin{array}{l}\text { I did not receive } \\
\text { any info }\end{array}$ & $\begin{array}{l}8 \\
(12.3)\end{array}$ & $\begin{array}{l}11 \\
(17.7)\end{array}$ \\
\hline & No, not at all & $\begin{array}{l}5 \\
(7.7)\end{array}$ & $3(4.8)$ \\
\hline & $\begin{array}{l}\text { I would rather not } \\
\text { say }\end{array}$ & $\begin{array}{l}2 \\
(3.1)\end{array}$ & $1(1.6)$ \\
\hline & Missing responses & $\begin{array}{l}0 \\
(0.0)\end{array}$ & $2(3.2)$ \\
\hline \multirow{6}{*}{ How useful did you find the survivorship information given to you? } & Very useful & $\begin{array}{l}27 \\
(41.5)\end{array}$ & $\begin{array}{l}35 \\
(56.5)\end{array}$ \\
\hline & Somewhat useful & $\begin{array}{l}24 \\
(36.9)\end{array}$ & $\begin{array}{l}13 \\
(21.0)\end{array}$ \\
\hline & Not useful & $\begin{array}{l}1 \\
(1.5)\end{array}$ & $0(0.0)$ \\
\hline & No opinion & $\begin{array}{l}2 \\
(3.1)\end{array}$ & $3(4.8)$ \\
\hline & $\begin{array}{l}\text { I would rather not } \\
\text { say }\end{array}$ & $\begin{array}{l}1 \\
(1.5)\end{array}$ & $1(1.6)$ \\
\hline & Missing responses & $\begin{array}{l}10 \\
(15.4)\end{array}$ & $\begin{array}{l}10 \\
(16.1)\end{array}$ \\
\hline \multirow{6}{*}{ How do you prefer to receive information about your survivorship? } & $\begin{array}{l}\text { Electronic form (e- } \\
\text { mail, website) }\end{array}$ & $\begin{array}{l}25 \\
(38.5)\end{array}$ & $\begin{array}{l}27 \\
(43.5)\end{array}$ \\
\hline & Paper form & $\begin{array}{l}23 \\
(35.4)\end{array}$ & $\begin{array}{l}21 \\
(33.9)\end{array}$ \\
\hline & Mobile app & $\begin{array}{l}6 \\
(9.2)\end{array}$ & $3(4.8)$ \\
\hline & $\begin{array}{l}\text { I do not want } \\
\text { information }\end{array}$ & $\begin{array}{l}2 \\
(3.1)\end{array}$ & $0(0.0)$ \\
\hline & $\begin{array}{l}\text { I would rather not } \\
\text { say }\end{array}$ & $\begin{array}{l}2 \\
(3.1)\end{array}$ & $1(1.6)$ \\
\hline & Missing responses & $\begin{array}{l}7 \\
(10.8)\end{array}$ & $\begin{array}{l}10 \\
(16.1)\end{array}$ \\
\hline \multirow{4}{*}{ How often do you miss an appointment in the survivorship clinic? } & Never & $\begin{array}{l}46 \\
(70.8)\end{array}$ & $\begin{array}{l}36 \\
(58.1)\end{array}$ \\
\hline & Rarely & $\begin{array}{l}9 \\
(13.8)\end{array}$ & $\begin{array}{l}13 \\
(21.0)\end{array}$ \\
\hline & Sometimes & $\begin{array}{l}4 \\
(6.2)\end{array}$ & $\begin{array}{l}7 \\
(11.3)\end{array}$ \\
\hline & Often & $\begin{array}{l}4 \\
(6.2)\end{array}$ & $2(3.2)$ \\
\hline
\end{tabular}




\section{Cureus}

\begin{tabular}{|c|c|c|c|}
\hline & $\begin{array}{l}\text { I would rather not } \\
\text { say }\end{array}$ & $\begin{array}{l}1 \\
(1.5)\end{array}$ & $1(1.6)$ \\
\hline & Missing responses & $\begin{array}{l}1 \\
(1.5)\end{array}$ & $3(4.8)$ \\
\hline \multirow{3}{*}{ Information about my cancer and how it was treated } & $\begin{array}{l}\text { I am getting the } \\
\text { right amount of } \\
\text { information }\end{array}$ & $\begin{array}{l}56 \\
(86.2)\end{array}$ & $\begin{array}{l}50 \\
(80.6)\end{array}$ \\
\hline & $\begin{array}{l}\text { I could use more } \\
\text { info }\end{array}$ & $\begin{array}{l}9 \\
(13.8)\end{array}$ & $\begin{array}{l}10 \\
(16.1)\end{array}$ \\
\hline & $\begin{array}{l}\text { I am getting too } \\
\text { much info }\end{array}$ & $\begin{array}{l}0 \\
(0.0)\end{array}$ & $2(3.2)$ \\
\hline \multirow{4}{*}{$\begin{array}{l}\text { Information about the healthcare system and how to talk with non-cancer doctors about my cancer diagnosis } \\
\text { and its treatment }\end{array}$} & $\begin{array}{l}\text { I am getting the } \\
\text { right amount of } \\
\text { information }\end{array}$ & $\begin{array}{l}45 \\
(69.2)\end{array}$ & $\begin{array}{l}39 \\
(62.9)\end{array}$ \\
\hline & $\begin{array}{l}\text { I could use more } \\
\text { info }\end{array}$ & $\begin{array}{l}17 \\
(26.2)\end{array}$ & $\begin{array}{l}18 \\
(29.0)\end{array}$ \\
\hline & $\begin{array}{l}\text { I am getting too } \\
\text { much info }\end{array}$ & $\begin{array}{l}2 \\
(3.1)\end{array}$ & $2(3.2)$ \\
\hline & $\begin{array}{l}\text { I would rather not } \\
\text { say }\end{array}$ & 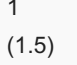 & $2(3.2)$ \\
\hline \multirow{4}{*}{$\begin{array}{l}\text { Information about health conditions resulting from my cancer treatment (such as pain, joint disease, heart } \\
\text { disease, development of second cancer, and neurocognitive changes such as decrease memory skills, } \\
\text { attention, math skills) }\end{array}$} & $\begin{array}{l}\text { I am getting the } \\
\text { right amount of } \\
\text { information }\end{array}$ & $\begin{array}{l}45 \\
(69.2)\end{array}$ & $\begin{array}{l}41 \\
(66.1)\end{array}$ \\
\hline & $\begin{array}{l}\text { I could use more } \\
\text { info }\end{array}$ & $\begin{array}{l}17 \\
(26.2)\end{array}$ & $\begin{array}{l}18 \\
(29.0)\end{array}$ \\
\hline & $\begin{array}{l}\text { I am getting too } \\
\text { much info }\end{array}$ & $\begin{array}{l}2 \\
(3.1)\end{array}$ & $3(4.8)$ \\
\hline & $\begin{array}{l}\text { I would rather not } \\
\text { say }\end{array}$ & $(1.5)$ & $0(0.0)$ \\
\hline \multirow{4}{*}{$\begin{array}{l}\text { Information or access to complimentary healthcare (such as the use of herbs or vitamins, the practice of yoga } \\
\text { or meditation, or receiving chiropractic or acupuncture treatment) }\end{array}$} & $\begin{array}{l}\text { I am getting the } \\
\text { right amount of } \\
\text { information }\end{array}$ & $\begin{array}{l}41 \\
(63.1)\end{array}$ & $\begin{array}{l}26 \\
(41.9)\end{array}$ \\
\hline & $\begin{array}{l}\text { I could use more } \\
\text { info }\end{array}$ & $\begin{array}{l}21 \\
(32.3)\end{array}$ & $\begin{array}{l}29 \\
(46.8)\end{array}$ \\
\hline & $\begin{array}{l}\text { I would rather not } \\
\text { say }\end{array}$ & $\begin{array}{l}1 \\
(1.5)\end{array}$ & $5(8.1)$ \\
\hline & Missing responses & $\begin{array}{l}2 \\
(3.1)\end{array}$ & $2(3.2)$ \\
\hline \multirow{5}{*}{ Learning about ways that I can help other patients or families } & $\begin{array}{l}\text { I am getting the } \\
\text { right amount of } \\
\text { information }\end{array}$ & $\begin{array}{l}26 \\
(40.0)\end{array}$ & $\begin{array}{l}24 \\
(38.7)\end{array}$ \\
\hline & $\begin{array}{l}\text { I could use more } \\
\text { info }\end{array}$ & $\begin{array}{l}31 \\
(47.7)\end{array}$ & $\begin{array}{l}30 \\
(48.4)\end{array}$ \\
\hline & $\begin{array}{l}\text { I am getting too } \\
\text { much info }\end{array}$ & $\begin{array}{l}1 \\
(1.5)\end{array}$ & $2(3.2)$ \\
\hline & $\begin{array}{l}\text { I would rather not } \\
\text { say }\end{array}$ & $\begin{array}{l}6 \\
(9.2)\end{array}$ & $4(6.5)$ \\
\hline & Missing responses & $\begin{array}{l}1 \\
(1.5)\end{array}$ & $2(3.2)$ \\
\hline
\end{tabular}

TABLE 3: Survey responses 


\section{Cureus}

Overall, there were high levels of satisfaction with the information the survivorship program provided to both group of participants. Overall, $78 \%$ of CACSs and PPCGs found the survivorship information useful, and $83 \%$ felt that they received the right amount of information about their cancer. The greatest difference between the two groups was the degree of importance for psychosocial support. PPCGs valued access to peer or survivor support groups, and information on where to seek help for managing feelings and learning coping strategies, $29 \%$ and $22.8 \%$ more, respectively, compared to the CACSs.

\section{Discussion}

The SC-PACS consortium is a unique collaboration of institutions representing varied models of healthcare, including freestanding children's hospitals, comprehensive cancer centers, managed care organizations, and private institutions. This heterogeneity leverages the strengths of each model, allows testing of interventions in varied settings, and ensures generalizability of results. The ultimate goal of the consortium is to develop a comprehensive survivorship care approach that addresses the most important needs of cancer survivors in our catchment area and promotes best practice interventions.

The demographic reach of the SC-PACS consortium consists of $62 \%$ self-reported Hispanic/Latino and $8 \%$ Asian. Because of the remarkable racial, ethnic, and sociodemographic diversity of southern California, the SC-PACS consortium stands uniquely in its position for serving and studying the minority population of cancer survivors. We recognize that given this diversity, it may be necessary to expand eligibility criteria of future study participants to patients who are neither completely proficient in English or Spanish and provide questionnaires that can be translated to other languages to promote participation.

Given that this was a feasibility study, our team acknowledged that our small sample size could be insufficient to show statistical significance. Although participants were recruited if they met the eligibility criteria for the study, purposeful selection methods were not rigorously adhered to, thus opening the possibility of selection bias. It is possible that we reached out to CACSs and PPCGs who would be willing to participate and potentially give a more positive feedback.

The importance of building a partnership with other nearby pediatric cancer survivorship centers has been recognized to promote collaborations for research endeavors and educational forums. Another pediatric cancer survivor consortium is the Consortium for New England Childhood Cancer Survivors (CONNECCS). This group was formed in 2011, consisting of 12 academic pediatric oncology institutions, serving a predominantly non-Hispanic white population in the New England region. The successful inception and publications of CONNECCS helped identify challenges and potential strategies for smaller, developing consortia [16-17].

The development of a Consortium Membership Agreement detailing core elements such as membership, data use, and administrative functions was integral to solidifying the consortium, as was establishing a core coordinating center. Moreover, successful completion of our pilot study demonstrates the commitment and ability of member institutions to execute multi-center survivorship studies. Not unexpectedly, some sites encountered administrative delays in obtaining IRB approval, which resulted in two sites being unable to approach 20 participants during the study period. Importantly, however, at both these sites, $100 \%$ of the CACSs or PPCGs who were approached did participate. Moving forward, the consortium intends to utilize a central IRB to reduce regulatory burden and facilitate study activation at member sites. Future plans are to expand the needs assessment survey in order to obtain broader representation of the survivor population at SC-PACS institutions. This may, in turn, inform strategies to improve cancer-specific education, delivery of treatment summary, and access to community resources including psychosocial needs for this demographically and socioeconomically diverse population.

\section{Conclusions}

Collaborations with nearby southern California pediatric cancer survivorship centers have enabled us to actively promote research endeavors and educational forums. Through the needs assessment study, we obtained data that specifically characterized our CACS population and their multiple survivorship needs. Future plans are to expand the needs assessment survey in order to obtain broader representation of the survivor population at SC-PACS institutions. This may, in turn, inform strategies to improve cancer-specific education, delivery of treatment summary, and access to community resources including psychosocial needs for this demographically and socioeconomically diverse population.

\section{Additional Information}

\section{Disclosures}


Human subjects: Consent was obtained or waived by all participants in this study. Children's Hospital of Orange County In-House IRB issued approval 1709102. DATE: October 13, 2017 TO: Carol Lin, MD FROM: Children's Hospital of Orange County In-House (CHOC IH) IRB IRB \#: 1709102 STUDY TITLE: 1709102 - SCPACS-001: Multi-institutional pilot study to assess the needs of childhood cancer survivors (CCS) followed in long-term survivorship clinics PROTOCOL: SPONSOR: Southern California Pediatric and Adolescent Cancer Survivorship Consortium (SC-PACS) STUDY STATUS: Active - Open to Enrollment IRBNET ID\#: 1130219-1 ACTION: APPROVED LEVEL OF REVIEW: Expedited Review, INITIAL MTG/ ACTION DATE: October 12, 2017 EXPIRATION DATE: October 11, 2018 REVIEW CATEGORY: Expedited Category \# 7 Thank you for your submission of New Project materials for this study on September 22, 2017. The Children's Hospital of Orange County In-House (CHOC IH) IRB has APPROVED your submission. This approval is based on an appropriate risk/benefit ratio and a project design wherein the risks have been minimized. All research must be conducted in accordance with this approved submission. Animal subjects: All authors have confirmed that this study did not involve animal subjects or tissue. Conflicts of interest: In compliance with the ICMJE uniform disclosure form, all authors declare the following: Payment/services info: All authors have declared that no financial support was received from any organization for the submitted work. Financial relationships: All authors have declared that they have no financial relationships at present or within the previous three years with any organizations that might have an interest in the submitted work. Other relationships: All authors have declared that there are no other relationships or activities that could appear to have influenced the submitted work.

\section{Acknowledgements}

We thank the SC-PACS institutions, clinical staff, patients, and caregivers who made this work possible. This work was funded by institutional resources within the SC-PACS consortium.

\section{References}

1. SEER Cancer Statistics Review (CSR) 1975-2017 . (2020). Accessed: January 8, 2022 : https://seer.cancer.gov/csr/1975_2017/.

2. Klipstein S, Fallat ME, Savelli S: Fertility preservation for pediatric and adolescent patients with cancer: medical and ethical considerations. Pediatrics. 2020, 145:e20193994. 10.1542/peds.2019-3994

3. Sadak KT, Neglia JP, Freyer DR, Harwood E: Identifying metrics of success for transitional care practices in childhood cancer survivorship: a qualitative study of survivorship providers. Pediatr Blood Cancer. 2017, 64:e26587. 10.1002/pbc.26587

4. Hudson MM, Ness KK, Gurney JG, et al.: Clinical ascertainment of health outcomes among adults treated for childhood cancer. JAMA. 2013, 309:2371-81. 10.1001/jama.2013.6296

5. Daly A, Lewis RW, Vangile K, Masker KW, Effinger KE, Meacham LR, Mertens AC: Survivor clinic attendance among pediatric- and adolescent-aged survivors of childhood cancer. J Cancer Surviv. 2019, 13:56-65. 10.1007/s11764-018-0727-3

6. Oeffinger KC, Hudson MM: Long-term complications following childhood and adolescent cancer: foundations for providing risk-based health care for survivors. CA Cancer J Clin. 2004, 54:208-36. 10.3322/canjclin.54.4.208

7. Nathan PC, Greenberg ML, Ness KK, et al.: Medical care in long-term survivors of childhood cancer: a report from the childhood cancer survivor study. J Clin Oncol. 2008, 26:4401-9. 10.1200/JCO.2008.16.9607

8. Aziz NM, Oeffinger KC, Brooks S, Turoff AJ: Comprehensive long-term follow-up programs for pediatric cancer survivors. Cancer. 2006, 107:841-8. 10.1002/cncr.22096

9. Berg CJ, Stratton E, Esiashvili N, Mertens A: Young adult cancer survivors' experience with cancer treatment and follow-up care and perceptions of barriers to engaging in recommended care. J Cancer Educ. 2016, 31:430-42. 10.1007/s13187-015-0853-9

10. Volerman A: Primary care of the childhood cancer survivor. Med Clin North Am. 2015, 99:1059-73. 10.1016/j.mcna.2015.05.005

11. Caplin DA, Smith KR, Ness KK, et al.: Effect of population socioeconomic and health system factors on medical care of childhood cancer survivors: a report from the childhood cancer survivor study. J Adolesc Young Adult Oncol. 2017, 6:74-82. 10.1089/jayao.2016.0016

12. Liu Q, Leisenring WM, Ness KK, Robison LL, Armstrong GT, Yasui Y, Bhatia S: Racial/ethnic differences in adverse outcomes among childhood cancer survivors: the childhood cancer survivor study. J Clin Oncol. 2016, 34:1634-43. 10.1200/JCO.2015.66.3567

13. Dixon SB, Li N, Yasui Y, et al.: Racial and ethnic disparities in neurocognitive, emotional, and quality-of-life outcomes in survivors of childhood cancer: a report from the Childhood Cancer Survivor Study. Cancer. 2019, 125:3666-77. 10.1002/cncr.32370

14. Leisenring WM, Mertens AC, Armstrong GT, et al.: Pediatric cancer survivorship research: experience of the Childhood Cancer Survivor Study. J Clin Oncol. 2009, 27:2319-27. 10.1200/JCO.2008.21.1813

15. Lee BA, Martin MJ, Matthews SA, Farrell CR: State-level changes in US racial and ethnic diversity, 1980 to 2015: a universal trend?. Demogr Res. 2017, 37:1031-48. 10.4054/DemRes.2017.37.33

16. Welch JJ, Kenney LB, Hirway P, et al.: Understanding predictors of continued long-term pediatric cancer care across the region: a report from the Consortium for New England Childhood Cancer Survivors. Pediatr Blood Cancer. 2017, 64:e26564. 10.1002/pbc.26564

17. Kenney LB, Bradeen H, Kadan-Lottick NS, Diller L, Homans A, Schwartz CL: The current status of follow-up services for childhood cancer survivors, are we meeting goals and expectations: a report from the Consortium for New England Childhood Cancer Survivors. Pediatr Blood Cancer. 2011, 57:1062-6. 10.1002/pbc. 22924 